\title{
Multifocal Motor Neuropathy With Anti-GM 1 Antibody: A Filipino Case Report
}

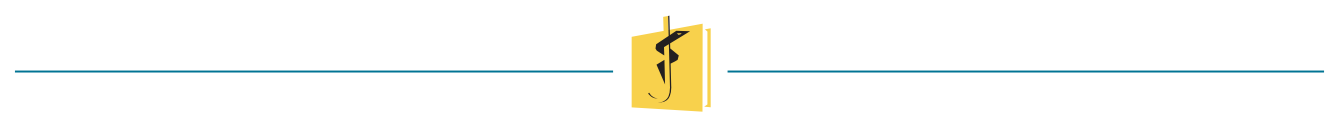

\author{
Gabriel Alejandro B. Baroque, MD, \\ Mary Lou Anne Y. Cabacang, MD, \\ Alejandro C. Baroque, MD
}

\section{ABSTRACT}

As far as we are aware of, we report the first documented case of a 51 -year-old Filipino female with multifocal motor neuropathy who presented with asymmetric weakness, unusually in the lower extremity, and confirmed with anti-GM1 antibody.

The treatment of intravenous immunoglobulin with a total dose of $2 \mathrm{~g} / \mathrm{kg}$ was initiated and repeated every two months with noticeable improvement based on electromyography and nerve conduction studies.

Apart from the unreported Filipino case of multifocal motor neuropathy substantiated by features in clinical, electrophysiologic, antibody testing and response to immunotherapy, the unique occurrence in a female and involving the lower extremity in this rare disorder deserve this present documentation. Multifocal motor neuropathy is seen more in males with a ratio of $2.7: 1$. It is described as a pure motor disease without sensory deficits and predominantly affects the upper extremities. The diagnosis for the disorder is supported by determination of ganglioside GM1 antibodies.

Keywords: multifocal motor neuropathy, case report

Gabriel Alejandro B. Baroque, MD gab_baroque51@yahoo.com

The Department of Neurology and Psychiatry University of Santo Tomas Hospital, Manila, Philippines

\section{INTRODUCTION}

The aim of the report is to document, for the first time, a case of multiple motor neuropathy $(M M N)$ in a Filipino who presented with asymmetric weakness and confirmed as having high anti-GM1 antibody titers coupled with abnormalities in electromyography (EMG) and nerve conduction studies (NCS).

$M M N$ is a rare disorder which was reported in 1986 by Chad, et al.[1] It is described as asymmetric motor weakness which is seen more on the upper extremities without sensory deficits.[2-8] This can be associated with fasciculations and cramping. [2] MMN has a prevalence of 0.6 per 100,000 individuals where males are predominantly involved with a ratio of $2.7: 1 .[6,9]$

Pestronk, et al., $[10,11]$ described $M M N$ to be associated with elevated ganglioside GM1 antibodies. [10] GM1 is localized in both the myelin and axolemma of peripheral nerves. The greatest population is found in the nodes of Ranvier. [6] This is the basis for the immunologic pathology associated with $M M N$.

The clinical criteria based on the European Federation of Neurological Societies/Peripheral Nerve Society are divided into three separate criteria. A) The core criteria; wherein the patient must manifest with: one, slowly progressive or stepwise progressive, focal, asymmetric limb weakness, that is, motor involvement in the motor nerve distribution of at least two nerves for more than one month (if symptoms and signs are present only in the distribution of one 
nerve, only a possible diagnosis can be made). Two, no objective sensory abnormalities except for minor vibration sense abnormalities in the lower limbs. B) Supportive clinical criteria are: 1. Predominant upper limb involvement, 2. Decreased or absent tendon reflexes in the affected limb, 3. Absence of cranial nerve involvement, 4. Cramps and fasciculations in the affected limb, 5. Response in terms of disability or muscle strength to immunomodulatory treatment. C) The exclusion criteria are: 1. Upper motor neuron signs, 2. Marked bulbar involvement, 3. Sensory impairment more marked than minor vibration loss in the lower limbs, 4. Diffuse symmetric weakness during the initial weeks. [4,6]

Electrophysiologic criteria for $M M N$ are as well divided into three categories: Definite motor conduction block, probable motor conduction block and normal sensory nerve conduction in the upper limb segments with conduction block. $[4,6]$

The other supportive criteria in the diagnosis of $M M N$ are elevated immunoglobulin $M(\lg M)$ anti-GM1 antibodies, an increased cerebrospinal fluid (CSF) protein $(<1 \mathrm{~g} / \mathrm{l})$, magnetic resonance imaging associated with a diffuse nerve swelling of the brachial plexus and clinical improvement following intravenous immunoglobulin (IVIg) treatment. $[4,6]$

\section{Patient Information:}

The patient is a 51-year-old female from the Philippines who presented with chronic left foot weakness. In 2009, the patient experienced left foot drop with no other accompanying symptoms such as numbness, back pain, paresthesias and bowel/ bladder incontinence. There was no history of any previous surgeries or trauma. During this time, EMG and NCS of the lower extremities were done which showed a left peroneal neuropathy and conduction block on the right ulnar nerve. She was advised to undergo exploration of the nerve; however, the patient declined and opted for medical management. She underwent a series of rehabilitations and was given Vitamin B complex tabs which afforded vague subjective improvement. In June 2018, a subsequent follow-up required a repeat EMG and NCS that now showed involvement of the left upper extremity.

\section{Clinical Findings:}

Upon a consult with us, there were no cognitive deficits and cranial nerve examination was not

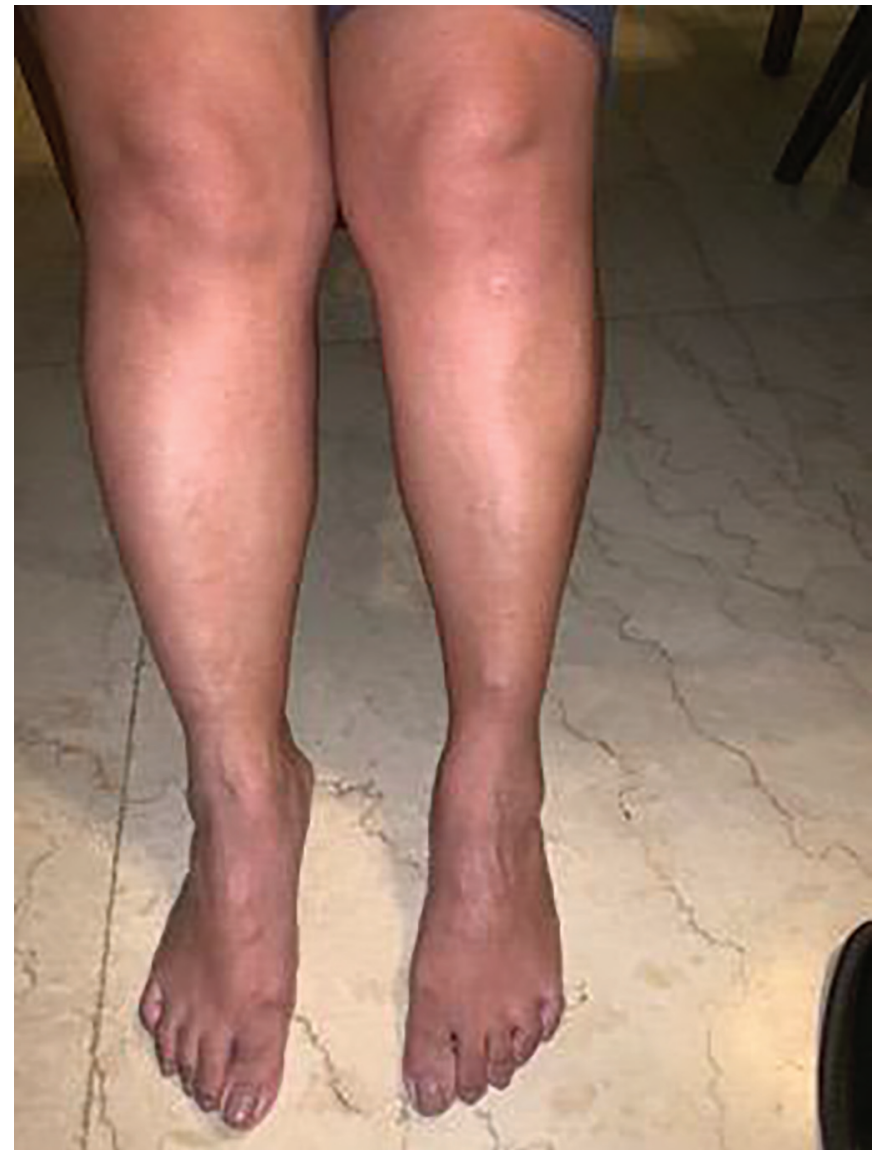

Figure 1. Showing atrophy of the left anterior tibialis muscle and loss of muscle bulk in the anterior compartment of the left leg (not seen is the atrophy of the abductor hallucis longus).

yielding. In particular, the eye movements were full and there was no tongue atrophy or fasciculations. Cerebellar examination was considered to be good. The patient's right wrist flexors, bilateral hip flexors and right dorsiflexion/plantar flexion had a manual motor testing of $4 / 5$. Her left knee flexion and left dorsiflexion/plantar flexion were noted to be weaker, compared to her other weak joint movers, having a manual motor testing of $2 / 5$. The patient also could not move her toes on her left foot. There was noted atrophy on her left tibialis anterior muscle and abductor hallucis longus (Figure 1). There were no sensory deficits and deep tendon reflexes were normoactive over both upper extremities and hyperactive over both lower extremities. Hoffman's and Babinski signs were not present and the jaw jerk was normal.

\section{Diagnostic Assessment:}

EMG and NCS done in November 2018 revealed that the right median and right ulnar nerves had conduction block at the level of the elbow up to the supraclavicular fossa and at the level of the axilla 

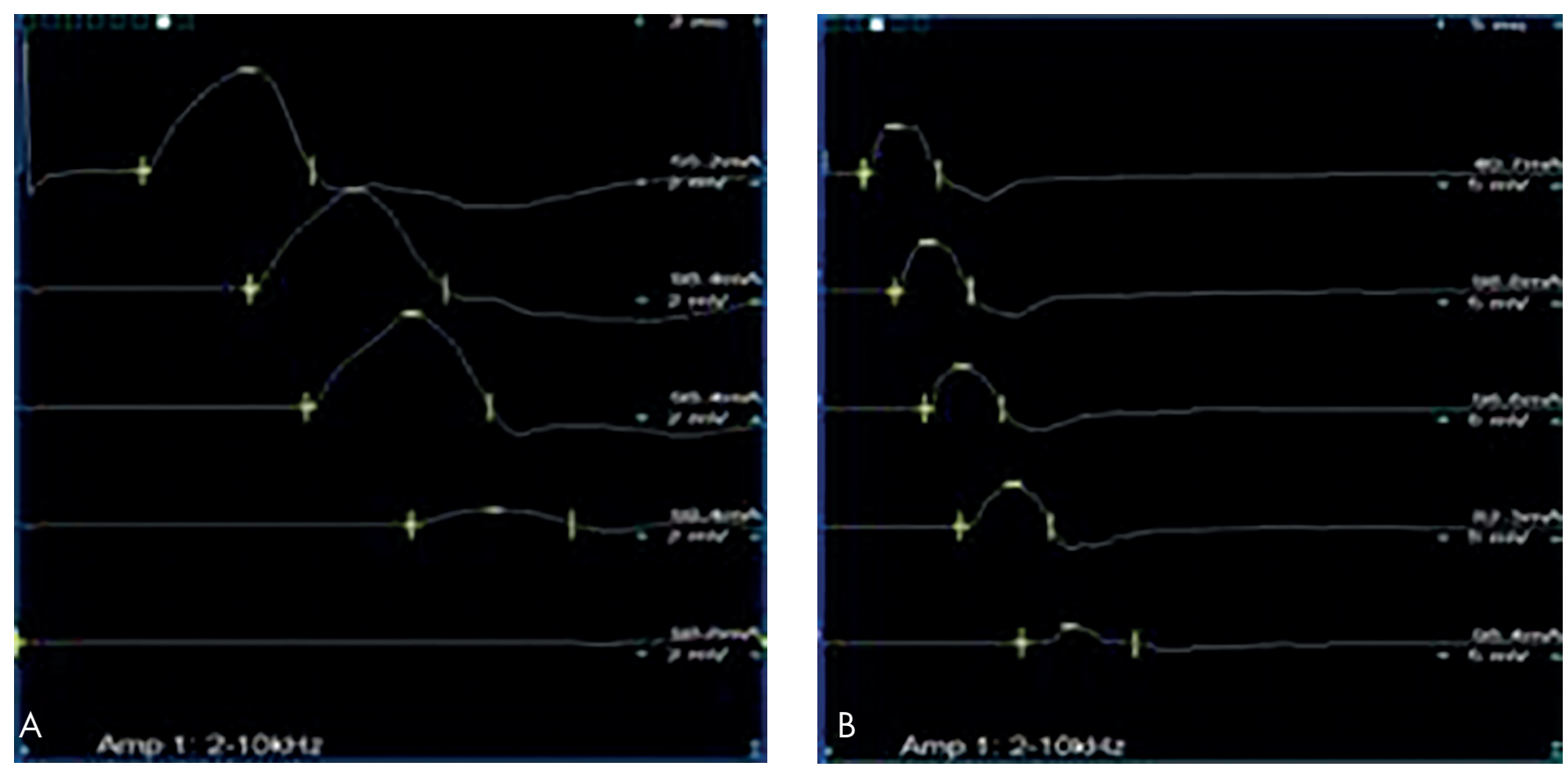

Figure 2. Serial nerve conduction studies (NCS) of the right ulnar nerve. Comparing the NCS done in November 2018 (A) and June 2019 (B), there is noted improvement of the CMAPs gleaned from proximal partial conduction blocks.
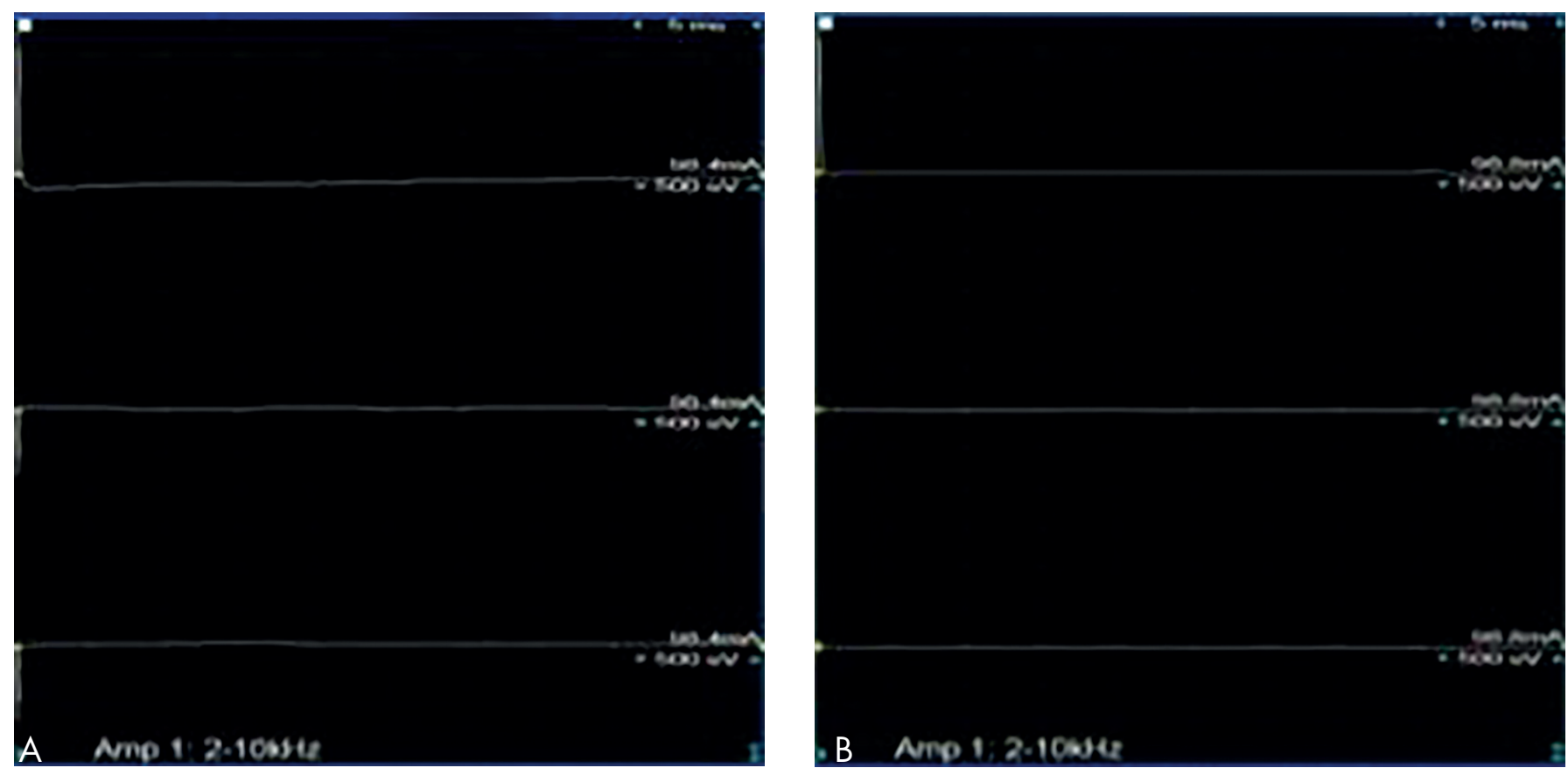

Figure 3. Serial nerve conduction studies (NCS) of the left peroneal motor nerve, showing absent evoked responses, comparing the NCS done in November 2018 (A) and June 2019 (B).

up to the supraclavicular fossa, respectively. There was reduced to absent, compound muscle action potential (CMAP) amplitudes on the bilateral peroneal and bilateral tibial nerves. There were normal $F$ wave responses on the right median, right ulnar and right tibial nerves. Sensory nerve conduction studies showed normal results. On needle examination, there was active denervation with chronic neurogenic changes on the muscles tested. These findings are consistent with MMN.(Figures 2-3).
Anti-GM1 antibody test was done in December 2018 which revealed a high titer of 1:12800 (Normal: <1:800).

\section{Intervention:}

Intravenous immunoglobulin (IVlg $2 \mathrm{~g} / \mathrm{kg}$ spread over five days) was administered in December 2018, and marked improvement of left knee flexion and plantar flexion were observed, yet plantar 
dorsiflexion remained weak. The patient now was also able to move her toes on her left foot.

\section{Followup and Outcomes:}

Follow-up IVlg same dose infusions were made in February and April 2019 and gradual motor strength improvements of the affected extremities continued.

A repeat electromyography-nerve conduction velocity (EMG-NCV) in June 2019 showed significant improvement in the ulnar motor NCS which previously showed conduction block on stimulation at the Erb's point, although with decreased CMAP amplitude. Left peroneal motor nerve conduction studies show increased CMAP amplitude. There was presence of F-wave response on the right ulnar nerve. On needle examination, there was absence of the previously noted spontaneous discharges in the right medial head of the gastrocnemius, left tibialis anterior, right first dorsal interosseous and right biceps brachii. In conclusion, there was improvement in the NCS and needle EMG (Figures 2-3).

However, after the hospital admission in April 2019, she was lost to followup until August 2019 wherein she complained of being unable to move her toes on the left foot with difficulty on plantar flexion. She still could not do dorsiflexion. Now, she was complaining of right hand weakness as well. Motor examination showed a finger abduction of $1 / 5$ on both hands, left knee extension of $2 / 5$, dorsiflexion and plantar flexion of the left foot $2 / 5$.

On admission in August 2019, she underwent infusion of IVlg for five days and on discharge she showed marked improvements in her motor examination with the finger abduction improving to $5 / 5$, left knee extension $5 / 5$, plantar flexion $5 / 5$ and dorsiflexion $2 / 5$. The patient was able to ambulate; however, the left foot drop remained.

\section{DISCUSSION}

We document for the first time, a rare case of $M M N$ occurring in a female from the Philippines. Uniquely, besides being a female, she clinically presented with asymmetric weakness of the lower extremity. The diagnosis of $M M N$ was based on electrophysiologic motor conduction blocks, sparing sensory nerves, presence of anti-GM1 serum antibody and favorable response to IVIg. There have been few cases of patients with $M M N$ that have been reported. Future local prevalence studies are highly recommended.
MMN is a rare disorder that can be easily misdiagnosed. Its prevalence is 0.6 per 100,000 individuals.[5] The onset of this disorder is generally before 50 years of age in $80 \%$ of the cases and it is predominantly seen in males with a ratio of 2.7:1. $[5,6,9]$ It presents as a progressive or stepwise asymmetric weakness without any sensory involvements. $[5,6]$ The upper extremities are usually affected in this disorder. Differential diagnoses for this disorder are chronic inflammatory demyelinating polyneuropathy (CIDP), particularly the pure motor form and Lewis-Sumner syndrome which has a sensory component. $M M N$ can be differentiated from CIDP by the apparent diffuse presentation of the latter, associated sensory abnormalities and hyporeflexia.[5] The diagnosis of $M M N$ can be done by careful history and physical examination of the patient. Clinical criteria supported by electrophysiologic studies and ganglioside GM1 antibodies should be considered in making such diagnoses. As for the treatment, IVlg infusion is the first line treatment for $M M N[4,5]$, and early treatment initiation may improve long-term impairment.[12]

\section{CONCLUSION}

We report for the first time a Filipino diagnosed with MMN showing compatible features of asymmetric weakness with atrophy, electrophysiologic motor conduction blocks, sparing sensory nerves and positive anti-GM1 antibody test. The unique occurrence in a Filipino female, mainly involving the lower extremity encouraged us to document this present case of $M M N$, rare at that.

\section{ETHICAL CONSIDERATIONS}

The investigators obtained approval of the University of Santo Tomas Hospital (USTH) Research Ethics Committee (REC) for the case report. CARE guidelines were carefully adhered to in the preparation of this manuscript.

\section{Declaration of Conflict of Interest}

The principal investigator was given authority by the team to present this case to avoid any potential competing interest in review or publication.

\section{Acknowledgment}

The authors acknowledge the invaluable advice of Dr. Imelda S. David and Dr. Raymond L. Rosales from the University of Santo Tomas contributing to the completion of this report. 


\section{REFERENCES}

1. Chad DA, Hammer K, Sargent J. Slow resolution of multifocal weakness and fasciculation: a reversible motor neuron syndrome. Neurology. 1986 Sep 1;36(9):1260.

2. Feldman EL, Bromberg MB, Albers JW, Pestronk A. Immunosuppressive treatment in multifocal motor neuropathy. Annals of Neurology: Official Journal of the American Neurological Association and the Child Neurology Society. 1991 Sep;30(3):397-401.

3. Olney RK, Lewis RA, Putnam TD, Campellone Jr JV. Consensus criteria for the diagnosis of multifocal motor neuropathy. Muscle \& Nerve: Official Journal of the American Association of Electrodiagnostic Medicine. 2003 Jan;27(1):1 17-21.

4. Van Asseldonk JT, Franssen $H$, Van den Berg-Vos RM, Wokke JH, Van den Berg LH. Multifocal motor neuropathy. The Lancet Neurology. 2005 May 1;4(5):309-19.

5. Joint Task Force of the EFNS and the PNS. European Federation of Neurological Societies/Peripheral Nerve Society guideline on management of multifocal motor neuropathy. Report of a joint task force of the European Federation of Neurological Societies and the Peripheral Nerve Societyfirst revision. Journal of the Peripheral Nervous System. 2010 Dec; 15(4):295-301.

6. Vlam L, van der Pol WL, Cats EA, Straver DC, Piepers S, Franssen $\mathrm{H}$, van den Berg LH. Multifocal motor neuropathy: diagnosis, pathogenesis and treatment strategies. Nature Reviews Neurology. 2012 Jan;8(1):48.

7. Lawson VH, Arnold WD. Multifocal motor neuropathy: a review of pathogenesis, diagnosis, and treatment. Neuropsychiatric Disease and Treatment. 2014;10:567.

8. Umapathi T, Hughes RA, Nobile-Orazio E, Léger JM. Immunosuppressant and immunomodulatory treatments for multifocal motor neuropathy. Cochrane Database of Systematic Reviews. 2015(3).
9. Beadon K, Guimarães-Costa R, Léger JM. Multifocal motor neuropathy. Current Opinion in Neurology. 2018 Oct;3 1 (5):559-64.

10. Pestronk A, Cornblath DR, llyas AA, Baba H, Quarles RH, Griffin JW, et al. A treatable multifocal motor neuropathy with antibodies to GMI ganglioside. Annals of Neurology: Official Journal of the American Neurological Association and the Child Neurology Society. 1988 Jul;24(1):73-8.

11. Pestronk A, Chaudhry V, Feldman EL, Griffin JW, Cornblath $\mathrm{DR}$, Denys EH, et al. Lower motor neuron syndromes defined by patterns of weakness, nerve conduction abnormalities, and high titers of antiglycolipid antibodies. Annals of Neurology: Official Journal of the American Neurological Association and the Child Neurology Society. 1990 Mar;27(3):316-26.

12. Al-Zuhairy A, Sindrup SH, Andersen H, Jakobsen J. A population-based and cross-sectional study of the long-term prognosis in multifocal motor neuropathy. Journal of the Peripheral Nervous System. 2019 Mar;24(1):64-71.

Open Access This article is licensed under a Creative Commons Attribution 4.0 International License, which permits use, sharing, adaptation, distribution and reproduction in any medium or format, as long as you give appropriate credit to the original author(s) and the source, provide a link to the Creative Commons license, and indicate if changes were made. The images or other third party material in this article are included in the article's Creative Commons license, unless indicated otherwise in a credit line to the material. If material is not included in the article's Creative Commons license and your intended use is not permitted by statutory regulation or exceeds the permitted use, you will need to obtain permission directly from the copyright holder. To view a copy of this license, visit http://creativecommons.org/licenses/by/4.0/. 